\title{
XANERAMÕJA PARAGETÃ
}

Textos:

\author{
Gilson Tenywaawi Tapirapé \\ Professor Pesquisador
}

Ilustrações:

Gilson Tenywaawi Tapirapé

Professor Pesquisador

Kaxowari’i Tapirapé

Professor Alfabetizador

\section{RESUMO}

Ã te’omara a'era mõ xexema’eaparepy, ãapa 'ã wekawo xerexekaxeka xema'eajpe maxywaatyãwa mõ. Te’omara ekwe aixagakãt Xaneramõja paragetã, ã rakaẽ xirot Topaxo agy Xyreni ne axaảyraxokare. Iexagakãta xerexywaaty, mảera tảe aoxekato xanereka, tarywa rereka ramõ konomi Takãra epexynawe'yma. Emanyn imaxywaatywo maragetã pitywera ma’eawete ramõ. Ipyrowo marama'eãra teomara xemaeãjpe, iapawo ite’omaãwa ramõ inoga ywyrape pe maragetã.

Palavras chave: Tarywa. Maragetã Xema'eãwa ramõ.

\section{XANERAMÕJA PARAGETÃ}

Iypy ramõ roõ rakaẽ irewee axyga.

A’e ro’o rakaẽ Xyreni aka irewee axyga ramõ takaripe. A'e ro’o rakãẽ tyke’yra "Topaxo"a’ym a’ywyrapãra iypa takaripe ranõ. 
A'e roõ rakaẽ Xyreni raty aa'opa mae,aakopaxi pae. A’e roõ rakaẽ amamyrõ akawo imemyra awyropiopi.

- Noripã 'ãwo api? Axãwo.

- Yni! E’i roõ rakaẽ irekawo ixope.

Awã wexe roõ ike’i aawo ranõ.

- Noripã 'ãwo api? Axãwo.

Awã wexe roõ ike’i aaworanõ.

- Nori pã 'ãwo api? Axãwo.

- Nori ãwo ney! Axãwo ixope.

-My tã a'e ramõ ika'i newi? E’i roõ irekawo ixope koxywera. Kwe roõ ia 'opa mae, axamamokamokãwo xe aakopaxi ne.

A’era roõ rakaẽ ia wowa we weakwa rewa ramõ takãrarakeni me.

- Xeropy, xeropy, xeropy! E'i’i roõ rakaẽ aawo.

A’eroõrakaẽ takeni me a’ym Topaxo, aywyrapãra iypa.

- He, he, he! E'i'i roõ rakaẽixope.

-Hããã, hããã, hããã!!! E’iixe’i roõ ahyjta aawo. Pe aawo imamyrõwo ranõ. A’e roõ rakaẽo rieranõ.

- Xeropy, xeropy, xeropy! Axãwo.

- He, he, he! E'i'i roõ ixope ranõ.

A'e ramõ rơõ rakaẽ aywyrapãraiywete, iakwa'akaixokaãwaramõ. A'e ro’o rakaẽaaranõ, maapãp.

- Xeropy, xeropy, xeropy! E'i'i roõ aawo ranõ.

- He, he, he! E'i'i ro'o ahyjta ixope aawo.

- Xak! Ixomanaka'i roõrakaẽ.

- Xoorok! Iexipikotoka’i roõ rakaẽ, aywyrapãra ramõ iapapyrape.

‘̃̃ roõ rakaẽ gyi rakwaema irekawo.

- Ky, ky, ky, ky, kyyyyyy!

- Hiiiiiie! E’i roó irekawo, takãra rakopiã imawãka.

A'e roõ rakaẽtowa axaj’a amarakãwo akawoee. Marygato mõta'e, nipaawiemigãwi.

Hahia he, hehe;

Hahie he, xera'yra! E'iroõiawyjxewomarakã.

-Maryn papa axyga, wa'yra re xei xaj’a akawo kyyy! E’i roõ ixope ixokaãra.

-Ma’era pã nerejxaki'i ememyraxewe hi! E’i roõa kawo a’ywyteray ramõwaty we. Kaaroka ramõ roõ emixe arywa (Ireweeaxyga). 'Ã ro’o rakaẽ apata, axokã roõ rakaẽ aawowaty. 
Kaaroka ramõ roõrakãẽ irapari pityga ranõ, awyropiopi iraãwo irekawo, ira’apokãjta, imawãka tãwa. A’ewi iraãwo takawyteripe ranõ. - Ky, ky, ky, ky, kyyyyyy!

- Hiiiiiie! Ika roõ gy.

'Ã roõ raka’ẽ a’ere irakewo takaripe. A’e kwera ropi roõ mi iraãimaapata irekawo tapi.

A’ere roõ rakaẽ gy ia awara'i imota, ta’yra axygepykawyma.

Amaenyn pane roõ rakaẽ Topaxo wa'yra re irekawo, namaatãakãri, awyripexe pane irekawo.

A’e roõ rakaẽ Xyreniaa 'yna takaripe, amaenyna ta'yra paawa re. Ixope xeroõ rakaẽ ipari, a’erooõ rakaẽi katykatye’ym apat awara’i pe. Kwewixe 'ota, i’awo ixowi takena, ake rapa xewi rea xãwo.

- Ho, ho, ho, ho!Axãwo. Ixomanakaa’egã ranõ, wa'yraxokaaweri mõ. Iejkwakotokaa'egã ranõ, ka iraawo takaripe. Emanyn roõ a'egã ireka ranõ, takaripe ira’apokãjta, a'ere tãwa imawãka.

A’eroõ rakã̃ amaxawãj ywykwera, Wyraxiga katy amõ, Araxã katy amõ. Araxãroõ rakaẽ pane a'egy.

A’ere roõ rakaẽ ia ataãra mõgy ranõ. A’era roõ xe rakaẽ a'egy ipari axaopi. A’eperoõ xe rakaẽ ixaxokã, marygato roõ xe rakaẽ ixawereka. 'Y peroõ e rakãẽ ixawera'ãri, naowi roõ rakã̃ wyrã.

A'era roõ mi Xyreni ikwãwi 'y ropi. A’e wi roõ 'ã xirekaxerexarywaramõ "Xaneramõja xãwa mõ". 

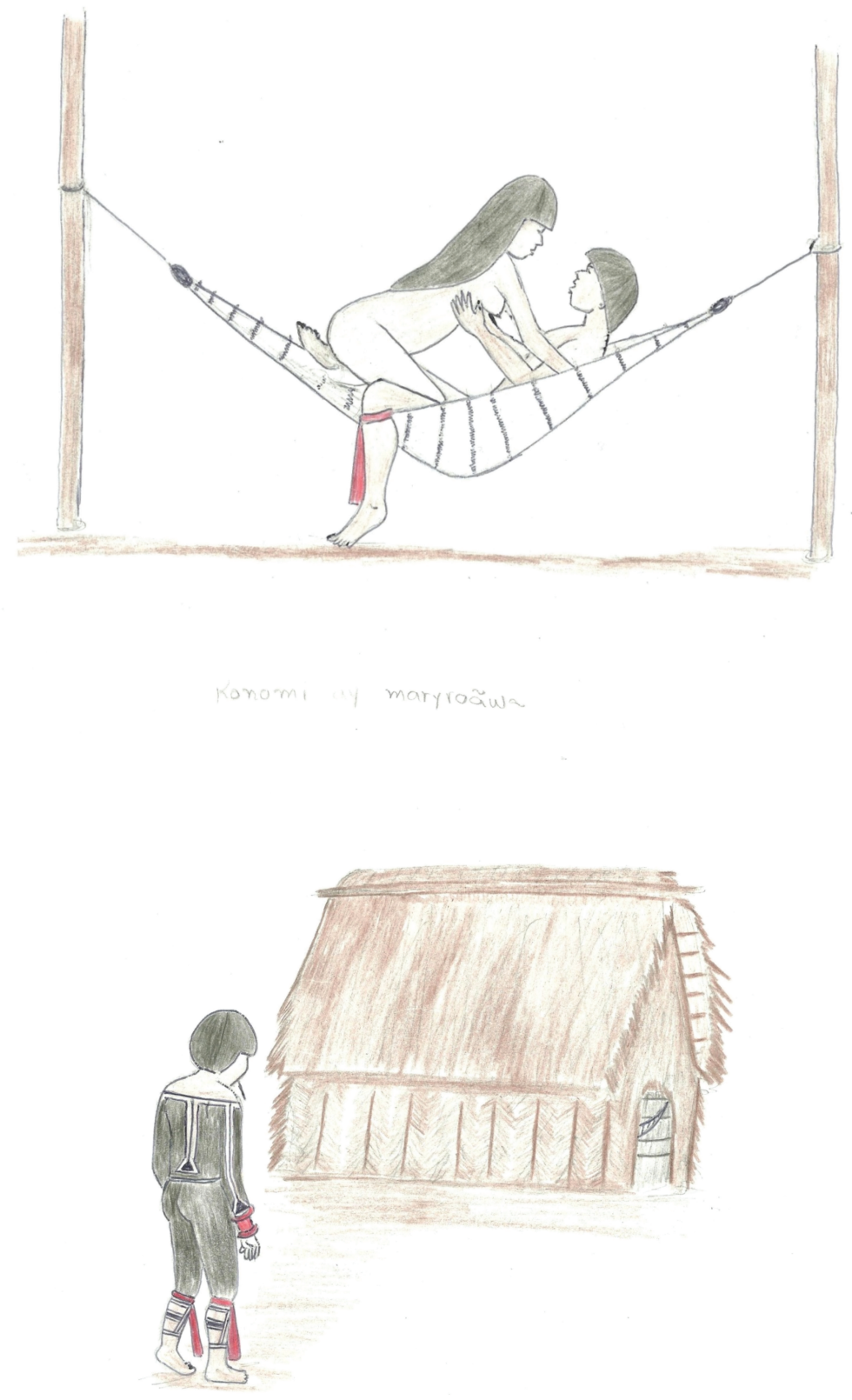
Takaripe Poraajtowa

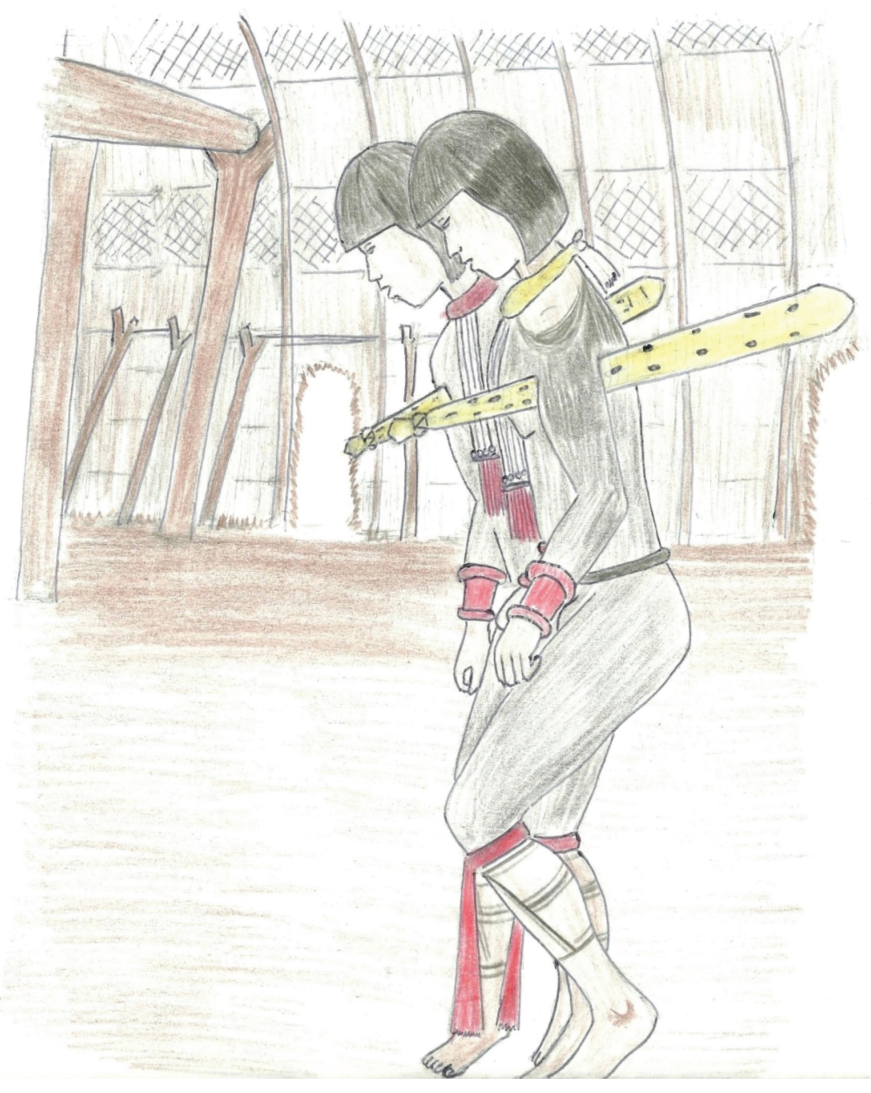



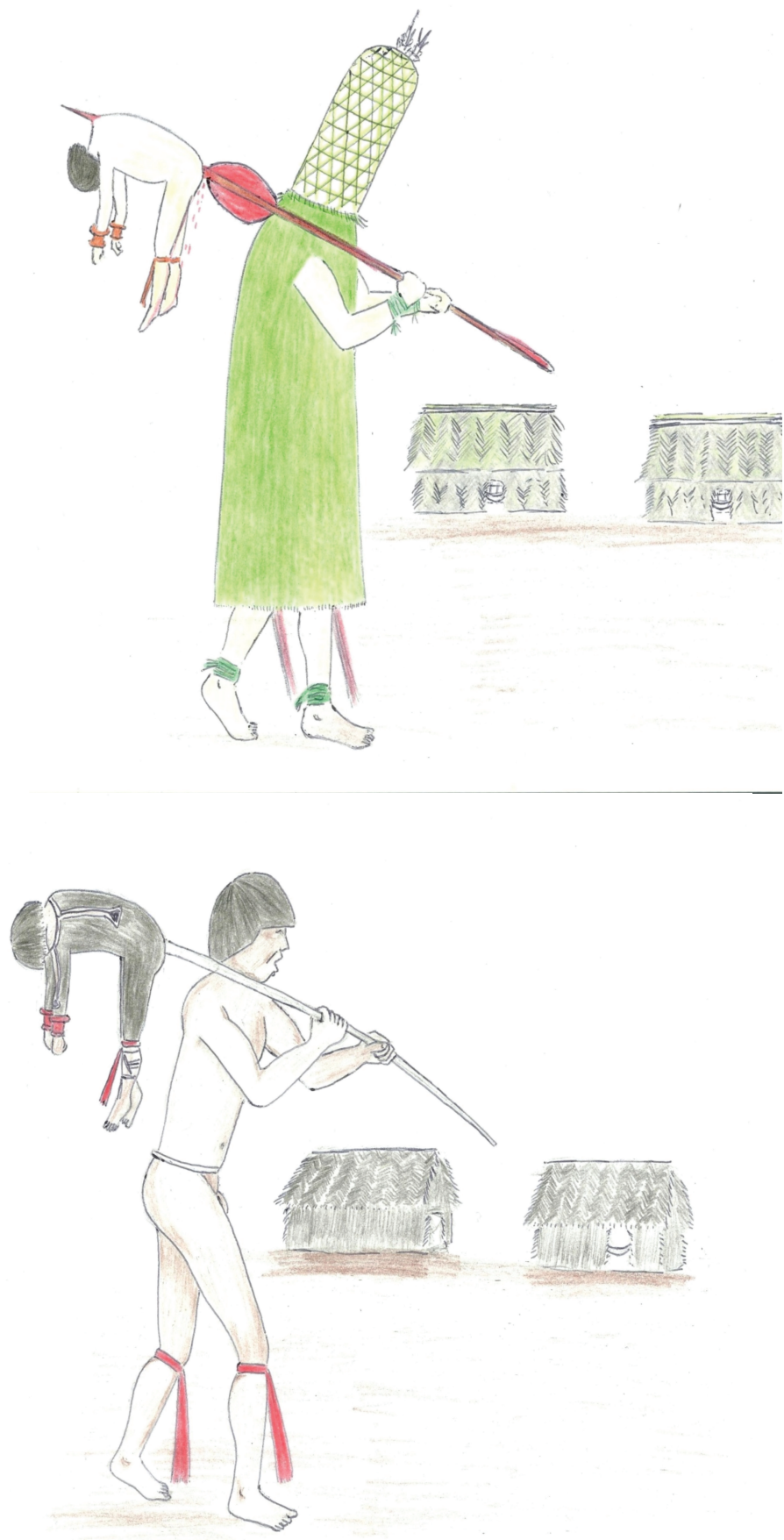

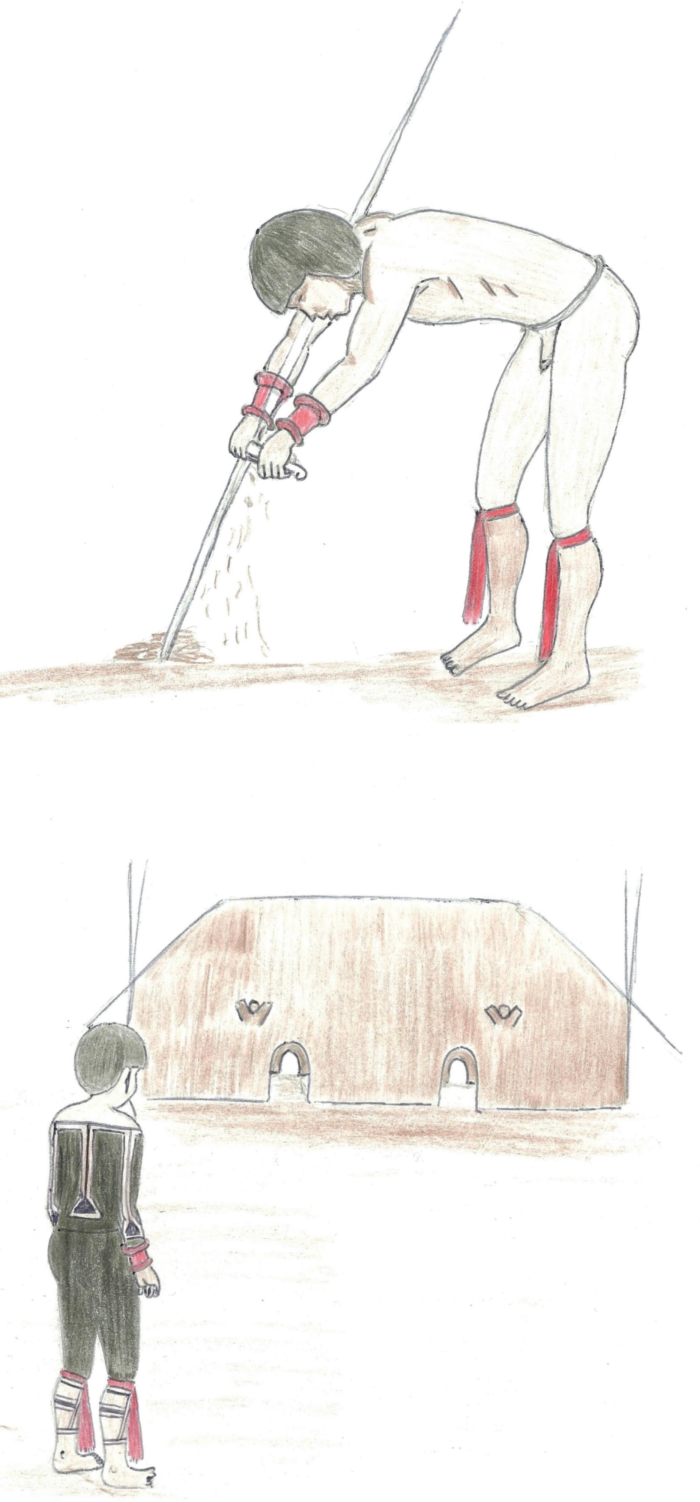


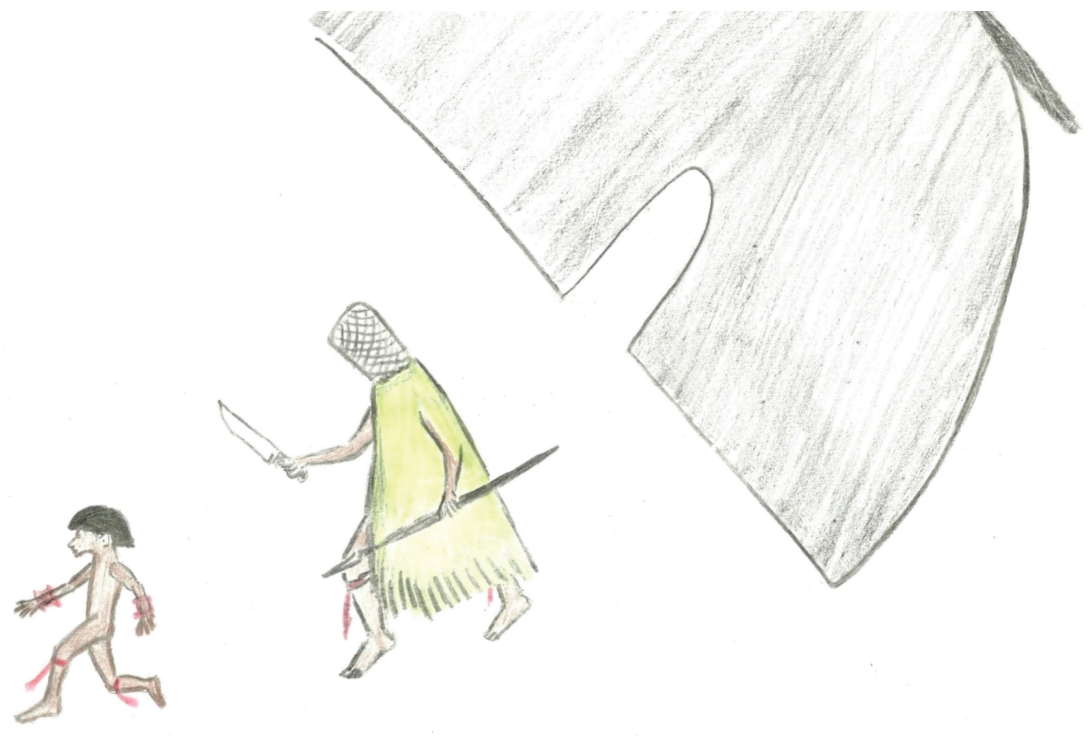

